International Mathematical Forum, Vol. 8, 2013, no. 8, 357 - 367

\title{
Small Zeros of Quadratic Forms Mod $P^{m}$
}

\author{
Ali H. Hakami \\ Department of Mathematics, Faculty of Science, Jazan University \\ P.O. Box 277, Jazan, Postal Code: 45142, Saudi Arabia \\ aalhakami@jazanu.edu.sa
}

\begin{abstract}
Let $Q(\mathbf{x})=Q\left(x_{1}, x_{2}, \ldots, x_{n}\right)$ be a quadratic form over $\mathbb{Z}, p$ be an odd prime, and $\Delta=\left((-1)^{n / 2} \operatorname{det} A_{Q} / p\right)$. A solution of the congruence $Q(\mathbf{x}) \equiv \mathbf{0}\left(\bmod p^{m}\right)$ is said to be a primitive solution if $p \nmid x_{i}$ for some $i$. We prove that if this congruence has a primitive solution then it has a primitive solution with $\|\mathbf{x}\| \leqslant \max \left\{6^{1 / n} p^{m[(1 / 2)+(1 / n)]}, 2^{2(n+1) /(n-2)} 3^{2 /(n-2)}\right\}$.
\end{abstract}

Mathematics Subject Classification: 11D79, 11E08, 11H50, 11 H55

Keywords: Quadratic forms, congruences, small solutions

\section{Introduction}

Let $Q(\mathbf{x})=Q\left(x_{1}, x_{2}, \ldots, x_{n}\right)=\sum_{1 \leqslant i \leqslant j \leqslant n} a_{i j} x_{i} x_{j}$ be a quadratic form with integer coefficients and $p$ be an odd prime. Set $\|\mathbf{x}\|=\max \left|x_{i}\right|$. When $n$ is even we let $\Delta_{p}(Q)=\left((-1)^{n / 2} \operatorname{det} A_{Q} / p\right)$ if $p \nmid \operatorname{det} A_{Q}$ and $\Delta_{p}(Q)=0$ if $p \mid \operatorname{det} A_{Q}$, where $(\cdot / p)$ denotes the Legendre-Jacobi symbol and $A_{Q}$ is the $n \times n$ defining matrix for $Q(\mathbf{x}) . Q(\mathbf{x})$ is called nonsingular $(\bmod p)$ if $p \nmid \operatorname{det} A_{Q}$.

Consider the congruence

$$
Q(\mathbf{x}) \equiv 0 \quad(\bmod M)
$$

where $M$ is a positive integer. The problem of finding a small solution of (1) means finding a nonzero integral solution $\mathbf{x}$ such that $\|\mathbf{x}\| \leq M^{\delta}$ for some positive constant $\delta<1$. The constant $\delta$ may depend on $n$, but not on $M$. Our interest in this paper is in finding a small primitive solution of (1) in the case where $M=p^{m}, m \geqslant 2$, a solution $\mathbf{x}$ with $\operatorname{gcd}\left(x_{1}, \ldots, x_{n}, M\right)=1$. A primitive solution is sought to rule out solutions of the type $p \mathbf{y}$ where $\mathbf{y}$ satisfies $Q(\mathbf{y}) \equiv 0(\bmod p)$. For the quadratic form $Q(x)=x_{1}^{2}+\cdots+x_{n}^{2}$, it is clear that any nonzero solution $\mathbf{x}$ of (1) must satisfy, max $\left|x_{i}\right| \geq \frac{1}{\sqrt{n}} M^{1 / 2}$. Thus $\delta=1 / 2$ is the best possible exponent for a small solution in general. 
Schinzel, Schlickewi and Schmidt [15] proved that (1) has a nonzero solution with $\|\mathbf{x}\|<M^{(1 / 2)+1 / 2(n-1)}$ for $n \geq 3$. Thus for any $\varepsilon>0$ we get a nonzero solution of (1) with $\|\mathbf{x}\|<M^{(1 / 2)+\varepsilon}$ provided $n$ is sufficiently large. We note that the solution obtained by their method is not necessarily a primitive solution. Indeed, when $M=p^{m}, m \geqslant 2$ they use a solution of the type $p \mathbf{y}$ with $Q(\mathbf{y}) \equiv 0(\bmod p)$.

Dealing with $M=p, p$ an odd prime, Heath-Brown [13] obtained a nonzero solution of (1) with $\|\mathbf{x}\| \ll p^{1 / 2} \log p$ for $n \geq 4$. His result was an improvement on the result of [15] in this case. Wang Yuan [16],[17] and [18] generalized Heath-Brown's work to all finite fields. Cochrane, in a sequence of papers [1], [2] and [3] improved this to $\|\mathbf{x}\|<\max \left\{2^{19} p^{1 / 2}, 2^{22} 10^{6}\right\}$. An improvement on Cochrane's result is given by Hakami [7, Theorem 1.3] and [10, Theorem 1] who obtained $\|\mathbf{x}\|<\min \left\{p^{2 / 3}, 2^{19} p^{1 / 2}\right\}$.

Hakami [8, Theorem 1] and [9, Theorem 1] respectively, generalized Cochrane's method to find a primitive solution of (1) with $\|\mathbf{x}\| \ll p$ for $n \geqslant 4$ when $M=p^{2}$ and with $\|\mathbf{x}\| \ll p^{3 / 2}$, for $n \geqslant 6$ when $M=p^{3}$ where $Q(\mathbf{y})$ is nonsingular $(\bmod p)$. Final Cochrane and Hakami $[6$, Theorem 1] proved that $\|x\| \leq p$ for $n>1$.

For $M=p q$ a product of two distinct primes, we find Heath-Brown [1] obtained $\|\mathbf{x}\| \ll M^{(1 / 2)+\varepsilon}$, for $n>4$ and $\varepsilon>0$. Later Cochrane [5] sharpened this result to $\|\mathbf{x}\| \ll M^{1 / 2}$, for $n>4$.

Our purpose in this paper is to generalize $(\bmod p)$ methods for obtaining a small primitive solution of

$$
Q(\mathbf{x}) \equiv 0 \quad\left(\bmod p^{m}\right)
$$

and more generally of obtaining a primitive solution in a box $\mathcal{B}$ with $|\mathcal{B}|$ sufficiently large.

Our main result is the following theorem.

Theorem 1.1 For any quadratic form $Q(\mathbf{x})$ with $n$ even, $n \geqslant 4$ and any odd prime power $p^{m}, m \geqslant 2$, there exists a primitive solution of (2) with

$$
\|\mathbf{x}\| \leqslant \max \left\{6^{1 / n} p^{m[(1 / 2)+(1 / n)]}, 2^{2(n+1) /(n-2)} 3^{2 /(n-2)}\right\} .
$$

Theorem 1.1 is an immediate consequence of Corollary 3.1. To prove Theorem 1.1 we shall use finite Fourier series over $\mathbb{Z}_{p^{m}}$, the ring of integers $\left(\bmod p^{m}\right)$.

\section{Basic Identities and Lemmas}

Henceforth we shall assume that $n$ is even, $p$ is an odd prime, and that $Q(\mathbf{x})$ is a nonsingular quadratic form $(\bmod p)$. Let $e_{p^{m}}(\alpha)=e^{2 \pi i \alpha / p^{m}}$. Let $V_{p^{m}}=$ 
$V_{p^{m}}(Q)$ be the set of zeros of $Q$ contained in $\mathbb{Z}_{p^{m}}^{n}$ and let $Q^{*}(\mathbf{y})$ be the quadratic form associated with inverse of the matrix for $Q(\bmod p)$. For $\mathbf{y} \in \mathbb{Z}_{p^{m}}^{n}$ set

$$
\phi\left(V_{p^{m}}, \mathbf{y}\right)= \begin{cases}\sum_{\mathbf{x} \in V} e_{p^{m}}(\mathbf{x} \cdot \mathbf{y}) & \text { for } \mathbf{y} \neq \mathbf{0} \\ \left|V_{p^{m}}\right|-p^{m(n-1)} & \text { for } \mathbf{y} \neq \mathbf{0}\end{cases}
$$

We abbreviate complete sums over $\mathbb{Z}_{p^{m}}^{n}$ and $\mathbb{Z}_{p}^{n}$ in the manner

$$
\sum_{\mathbf{x}}=\sum_{\mathbf{x} \bmod p^{m}}=\sum_{x_{1}=1}^{p^{m}} \cdots \sum_{x_{n}=1}^{p^{m}}, \quad \sum_{\mathbf{x} \bmod p}=\sum_{x_{1}=1}^{p} \cdots \sum_{x_{n}=1}^{p}
$$

The following lemma gives us a formula for $\phi(V, \mathbf{y})$.

Lemma 2.1 Let $n$ be is even positive integer. For $\mathbf{y} \in \mathbb{Z}^{n}$, put $\mathbf{y}^{\prime}=p^{-j} \mathbf{y}$ in case $p \mid \mathbf{y}$. Then

$$
\phi(V, \mathbf{y})=p^{(m n / 2)-m} \sum_{\substack{j=0 \\ p^{j} \mid y_{i} \text { for all } i}}^{m-1} \delta_{j} p^{j n / 2} \omega_{j}\left(\mathbf{y}^{\prime}\right),
$$

where

$$
\delta_{j}= \begin{cases}1 & \text { if } m-j \text { is even } \\ \Delta & \text { if } m-j \text { is odd }\end{cases}
$$

and

$$
\omega_{j}\left(\mathbf{y}^{\prime}\right)= \begin{cases}p^{m-j}-p^{m-j-1}, & p^{m-j} \mid Q^{*}\left(\mathbf{y}^{\prime}\right), \\ -p^{m-j-1}, & p^{m-j-1} \| Q^{*}\left(\mathbf{y}^{\prime}\right), \\ 0, & p^{m-j-1} \nmid Q^{*}\left(\mathbf{y}^{\prime}\right) .\end{cases}
$$

The proof of Lemma 2.1 is given (with some work) in Carlitz [12], and in complete detail in [11, Theorem 1].

Let $\alpha(\mathbf{x})$ be a complex valued function defined on $\mathbb{Z}_{p^{m}}^{n}$ with Fourier expansion $\alpha(\mathbf{x})=\sum_{\mathbf{y}} a(\mathbf{y}) e_{p^{m}}(\mathbf{x} \cdot \mathbf{y})$ where $a(\mathbf{y})=p^{-m n} \sum_{\mathbf{x}} \alpha(\mathbf{x}) e_{p^{m}}(-\mathbf{x} \cdot \mathbf{y})$. Then by definition of $\phi(V, \mathbf{y})$, 


$$
\begin{aligned}
\sum_{\mathbf{x} \in V_{p^{m}}} \alpha(\mathbf{x}) & =a(\mathbf{0})\left|V_{p^{m}}\right|+\sum_{\mathbf{y} \neq \mathbf{0}} a(\mathbf{y}) \sum_{\mathbf{x} \in V_{p^{m}}} e_{p^{m}}(\mathbf{y} \cdot \mathbf{x}) \\
& =a(\mathbf{0})\left|V_{p^{m}}\right|+\sum_{\mathbf{y} \neq \mathbf{0}} a(\mathbf{y}) \phi(V, \mathbf{y}) \\
& =a(\mathbf{0})\left[\phi(V, \mathbf{0})+p^{m(n-1)}\right]+\sum_{\mathbf{y} \neq \mathbf{0}} a(\mathbf{y}) \phi(V, \mathbf{y}) \\
& =a(\mathbf{0}) p^{m(n-1)}+\sum_{\mathbf{y}} a(\mathbf{y}) \phi(V, \mathbf{y}) \\
& =p^{-m n} \sum_{\mathbf{x}} \alpha(\mathbf{x}) p^{m(n-1)}+\sum_{\mathbf{y}} a(\mathbf{y}) \phi(V, \mathbf{y})
\end{aligned}
$$

Thus by Lemma 2.1,

$$
\begin{aligned}
\sum_{\mathbf{x} \in V_{p} m} \alpha(\mathbf{x}) & =p^{-m} \sum_{\mathbf{x}} \alpha(\mathbf{x})+p^{(m n / 2)-m} \sum_{\mathbf{y}} a(\mathbf{y}) \sum_{\substack{j=0 \\
p^{j} \mid y_{i} \text { for all } i}}^{m-1} \delta_{j} p^{j n / 2} \omega_{j}\left(\mathbf{y}^{\prime}\right) \\
& =p^{-m} \sum_{\mathbf{x}} \alpha(\mathbf{x})+p^{(m n / 2)-m} \sum_{j=0}^{m-1} \delta_{j} p^{j n / 2} \sum_{\substack{\mathbf{y} \\
p^{j} \mid y_{i} \text { for all } i}} a(\mathbf{y}) \omega_{j}\left(\mathbf{y}^{\prime}\right) .
\end{aligned}
$$

But by noticing that the inner sum $\sum_{\mathbf{y}} a\left(p^{j} \mathbf{y}^{\prime}\right) \omega_{j}\left(y^{\prime}\right)$ can be written

$$
\begin{aligned}
& \sum_{\substack{\mathbf{y} \\
p^{j} \mid y_{i} \text { for all } i}} a(\mathbf{y}) \omega_{j}\left(\mathbf{y}^{\prime}\right)=\sum_{y^{\prime}\left(\bmod p^{m-j}\right)} a\left(p^{j} \mathbf{y}^{\prime}\right) \omega_{j}\left(\mathbf{y}^{\prime}\right) \\
= & \sum_{\substack{\mathbf{y}^{\prime} \\
p^{m-j} \mid Q^{*}\left(\mathbf{y}^{\prime}\right)}} a\left(p^{j} \mathbf{y}^{\prime}\right)\left(p^{m-j}-p^{m-j-1}\right)-\sum_{\substack{\mathbf{y}^{\prime} \\
p^{m-j-1} \| Q^{*}\left(\mathbf{y}^{\prime}\right)}} a\left(p^{j} \mathbf{y}^{\prime}\right) p^{m-j-1} \\
= & \sum_{\substack{\mathbf{y}^{\prime} \\
p^{m-j} \mid Q^{*}\left(\mathbf{y}^{\prime}\right)}} a\left(p^{j} \mathbf{y}^{\prime}\right) p^{m-j}-p^{m-j-1} \sum_{\substack{\mathbf{y}^{\prime} \\
p^{m-j-1} \mid Q^{*}\left(\mathbf{y}^{\prime}\right)}} a\left(p^{j} \mathbf{y}^{\prime}\right),
\end{aligned}
$$

we obtain,

Lemma 2.2 (The fundamental identity) For any complex valued $\alpha(\mathbf{x})$ 
as given above

$$
\begin{aligned}
& \sum_{\mathbf{x} \in V_{p} m} \alpha(\mathbf{x})=p^{-m} \sum_{\mathbf{x}\left(\bmod p^{m}\right)} \alpha(\mathbf{x}) \\
& +p^{m n / 2} \sum_{j=0}^{m-1} p^{j n / 2} \delta_{j}\left(p^{-j} \sum_{\substack{y_{i}^{\prime}=1 \\
p^{m-j} \mid Q^{*}\left(\mathbf{y}^{\prime}\right)}}^{p^{m-j}} a\left(p^{j} \mathbf{y}^{\prime}\right)-p^{-j-1} \sum_{\substack{y_{i}^{\prime}=1 \\
p^{m-j-1} \mid Q^{*}\left(\mathbf{y}^{\prime}\right)}}^{p^{m-j}} a\left(p^{j} \mathbf{y}^{\prime}\right)\right),
\end{aligned}
$$

where $\delta_{j}$ as we defined in (3): $\delta_{j}=1$ when $m-j$ is even and $\delta_{j}=\Delta$ if $m-j$ is odd.

\section{Proof of Theorem 1.1 when $\Delta=+1$}

Let $\mathcal{B}$ be the box of points in $\mathbb{Z}^{n}$ given by

$$
\mathcal{B}=\left\{\mathbf{x} \in \mathbb{Z}^{n} \mid a_{i} \leqslant x_{i}<a_{i}+B_{i}, 1 \leqslant i \leqslant n\right\},
$$

where $a_{i}, B_{i} \in \mathbb{Z}$ and $1 \leqslant B_{i} \leqslant p^{m}, \quad 1 \leqslant i \leqslant n$. Then $|\mathcal{B}|=\prod_{i=1}^{n} B_{i}$, the cardinality of $\mathcal{B}$. View the box $\mathcal{B}$ in (4) as a subset of $\mathbb{Z}_{p^{m}}^{n}$ and let $\alpha(\mathbf{x})=\chi_{\mathcal{B}} * \chi_{\mathcal{B}}$ with Fourier expansion $\alpha(\mathbf{y})=\sum_{\mathbf{y}} a(\mathbf{y}) e_{p^{m}}(\mathbf{x} \cdot \mathbf{y})$. Thus for any $\mathbf{y} \in \mathbb{Z}_{p^{m}}^{n}$,

$$
\left|a_{(\mathbf{y})}\right|=p^{-m n} \prod_{i=1}^{n}\left|\frac{\sin ^{2} \pi B_{i} y_{i} / p^{m}}{\sin ^{2} \pi y_{i} / p^{m}}\right|,
$$

where the term in the product is taken to be $m_{i}$ if $y_{i}=0$. In particular

$$
a(\mathbf{y}) \leqslant p^{-m n} \prod_{i=1}^{n} \min \left\{B_{i}^{2},\left(\frac{p^{m}}{2 y_{i}}\right)^{2}\right\}
$$

Consider the congruence (2):

$$
Q(\mathbf{x}) \equiv 0 \quad\left(\bmod p^{m}\right) .
$$

For later reference, we construct the following lemma which help us on estimating the sum $\sum_{y_{i}=1}^{p^{m-j}} a\left(p^{j} \mathbf{y}\right)$.

Lemma 3.1 Let $\mathcal{B}$ be any box of type (4) and $\alpha(\mathbf{x})=\chi_{\mathcal{B}} * \chi_{\mathcal{B}}(\mathbf{x})$. Then we have

$$
\sum_{y_{i}=1}^{p^{m-j}} a\left(p^{j} \mathbf{y}\right) \leqslant \frac{|\mathcal{B}|}{p^{j n}} \prod_{B_{i} \geqslant p^{m-j}} \frac{2 B_{i}}{p^{m-j}}
$$


Proof. First,

$$
\begin{aligned}
\sum_{y_{i}=1}^{p^{m-j}} a\left(p^{j} \mathbf{y}\right) & =\sum_{\mathbf{y}} \sum_{x_{i}=\mathbf{1}}^{p^{m}} \frac{1}{p^{m}} \alpha(\mathbf{x}) e_{p^{m}}\left(-\mathbf{x} \cdot p^{j} \mathbf{y}\right) \\
& =\sum_{x_{i}=1}^{p^{m}} \frac{1}{p^{m n}} \alpha(\mathbf{x}) \sum_{y_{i}} e_{p^{m}}\left(-\mathbf{x} \cdot p^{j} \mathbf{y}\right) \\
& =\sum_{x_{i}=1}^{p^{m}} \frac{1}{p^{m n}} \alpha(\mathbf{x}) \sum_{y_{i}=1}^{p^{m-j}} e_{p^{m-j}}(-\mathbf{x} \cdot \mathbf{y}) \\
& =\sum_{x_{i}=1}^{p^{m}} p^{-m n} \alpha(\mathbf{x}) p^{(m-j) n} \\
& =p^{-j n} \sum_{x_{i}=1}^{p^{m}} \alpha(\mathbf{x}) \\
& =p^{-j n} \sum_{\mathbf{x} \in \mathcal{B}} \sum_{\mathbf{v} \in \mathcal{B}} 1 \\
\leqslant & p^{-j n} \prod_{i=1}^{n} B_{i}\left(\left[\frac{B_{i}}{p^{m-j}}\right]+1\right)
\end{aligned}
$$

To verify the last inequality in (5) we count the number of solutions of the congruence

$$
\mathbf{u}+\mathbf{v} \equiv 0 \quad\left(\bmod p^{m-j}\right),
$$

with $\mathbf{u}, \mathbf{v} \in \mathcal{B}$. In fact for each choice of $\mathbf{v}$, there are at most $\prod_{i=1}^{n}\left(\left[B_{i} / p^{m-j}\right]+\right.$ 1) ch-oices $\mathbf{u}$. Thus the total number of solutions is less than or equal to $\prod_{i=1}^{n}\left(\left[B_{i} / p^{m-j}\right]+1\right)$. Hence it follows from $(5)$,

$$
\sum_{y_{i}=1}^{p^{2}} a(p \mathbf{y}) \leqslant p^{-j n} \prod_{i=1}^{n} B_{i}\left(\left[\frac{B_{i}}{p^{m-j}}\right]+1\right) .
$$

We may split the product in (6) such that

$$
\prod_{i=1}^{n} B_{i}\left(\left[\frac{B_{i}}{p^{m-j}}\right]+1\right)=\prod_{B_{i}<p^{m-j}} B_{i} \prod_{B_{i} \geqslant p^{m-j}} B_{i}\left(\frac{2 B_{i}}{p^{m-j}}\right) .
$$

Then by this equality we therefore have

$$
\sum_{y_{i}=1}^{p^{m-j}} a\left(p^{j} \mathbf{y}\right) \leqslant \frac{|\mathcal{B}|}{p^{j n}} \prod_{B_{i} \geqslant p^{m-j}} \frac{2 B_{i}}{p^{m-j}}
$$


Our proof is complete.

Now we can proceed to estimate the error term in the fundamental identity which is given by

Error $=p^{m n / 2} \sum_{j=0}^{m-1} p^{j n / 2} \delta_{j}\left(p^{-j} \sum_{\substack{y_{i}^{\prime}=1 \\ p^{m-j} \mid Q^{*}\left(\mathbf{y}^{\prime}\right)}}^{p^{m-j}} a\left(p^{j} \mathbf{y}^{\prime}\right)-p^{-j-1} \sum_{\substack{y_{i}^{\prime}=1 \\ p^{m-j-1} \mid Q^{*}\left(\mathbf{y}^{\prime}\right)}}^{p^{m-j}} a\left(p^{j} \mathbf{y}^{\prime}\right)\right)$.

By Lemma 3.1 we get

$$
\mid \text { Error }\left|\leqslant p^{m n / 2} \sum_{j=0}^{m-1} p^{j n / 2}\right| \sum_{y_{i}=1}^{p^{m-j}} \varphi(\mathbf{y}) a\left(p^{j} \mathbf{y}^{\prime}\right) \mid
$$

where

$$
\varphi(\mathbf{y})=\left\{\begin{array}{ccc}
p^{j}-p^{-j-1} & \text { if } & p^{m-j} \mid Q^{*}(\mathbf{y}) \\
-p^{-j-1} & \text { if } & p^{m-j-1} \| Q^{*}(\mathbf{y})
\end{array}\right.
$$

Continuing from (7),

$$
\begin{aligned}
\mid \text { Error } \mid & \leqslant p^{m n / 2} \sum_{j=0}^{m-1} p^{(j n / 2)-j} \frac{|\mathcal{B}|}{p^{j n}} \prod_{B_{i} \geqslant p^{m-j}} \frac{2 B_{i}}{p^{m-j}} \\
& =p^{m n / 2}|\mathcal{B}| \sum_{j=0}^{m-1} \frac{1}{p^{(j n / 2)+j}} \prod_{B_{i} \geqslant p^{m-j}} \frac{2 B_{i}}{p^{m-j}} .
\end{aligned}
$$

We now restrict our attention to the case where $\mathcal{B}$ is cube, that is, $B_{i}=B$, $1 \leqslant i \leqslant n$. Say $p^{j_{0}} \leqslant B<p^{j_{0}+1}$ for some $j_{0} \in \mathbb{N}, 1 \leqslant j_{0}<m$. Then

$$
B \geqslant p^{m-j} \Longleftrightarrow m-j \leqslant j_{0} \Longleftrightarrow j \geqslant m-j_{0} .
$$

Continuing from (8), we get

$$
\begin{aligned}
\mid \text { Error } \mid & \leqslant p^{m n / 2}|\mathcal{B}| \sum_{j=m-j_{0}}^{m-1} \frac{2^{n}|\mathcal{B}|}{p^{(j n / 2)+j} p^{n m-n j}}+p^{m n / 2}|\mathcal{B}| \sum_{j=0}^{m-j_{0}-1} \frac{1}{p^{(j n / 2)+j}} \\
& =\frac{2^{n}|\mathcal{B}|^{2}}{p^{m n / 2}} \sum_{j=m-j_{0}}^{m-1} p^{(n / 2) j-j}+p^{m n / 2}|\mathcal{B}| \sum_{j=0}^{m-j_{0}-1} \frac{1}{p^{(j n / 2)+j}} \\
& <\frac{2^{n}|\mathcal{B}|^{2}}{p^{m n / 2}} p^{[(n / 2)-1](m-1)} \cdot 2+p^{m n / 2}|\mathcal{B}| \cdot 2
\end{aligned}
$$




$$
\begin{aligned}
& =2^{n+1}|\mathcal{B}|^{2} p^{-m-(n / 2)+1}+2 p^{m n / 2}|\mathcal{B}| \\
& =\frac{2^{n+1}|\mathcal{B}|^{2}}{p^{m+(n / 2)-1}}+2 p^{m n / 2}|\mathcal{B}|
\end{aligned}
$$

Proposition 3.1 Suppose that $m \geqslant 2, n \geqslant 4, n$ even. Then for any cube $\mathcal{B}$ centered at the origin,

$$
\sum_{\mathbf{x} \in V} \alpha(\mathbf{x}) \geqslant \frac{|\mathcal{B}|^{2}}{p^{m}}-\mid \text { Error } \mid,
$$

where

$$
\mid \text { Error } \mid \leqslant \underbrace{\frac{2^{n+1}|\mathcal{B}|^{2}}{p^{m+(n / 2)-1}}}_{\text {Error } 1}+\underbrace{2 p^{m n / 2}|\mathcal{B}|}_{\text {Error } 2} .
$$

We compare Error 1 and Error 2 in Proposition 3.1 to the main term $|\mathcal{B}|^{2} / p^{m}$. In order to make the left-hand side positive, we make each error term less than $1 / 3$ of the main term. For the error term Error 1, we need

$$
\begin{gathered}
\frac{2^{n+1}|\mathcal{B}|^{2}}{p^{m+(n / 2)-1}}<\frac{1}{3} \frac{|\mathcal{B}|^{2}}{p^{m}} \Longleftrightarrow p^{(n / 2)-1}>3.2^{n+1} \\
\Longleftrightarrow \quad p>2^{2(n+1) /(n-2)} \cdot 3^{2 /(n-2)} .
\end{gathered}
$$

For the error term Error 2,

$$
\begin{aligned}
2 p^{m n / 2}|\mathcal{B}| & <\frac{1}{3} \frac{|\mathcal{B}|^{2}}{p^{m}} \Longleftrightarrow|\mathcal{B}|>3.2 p^{(m n / 2)+m} \\
& \Longleftrightarrow B>6^{1 / n} p^{(m / 2)+(m / n)} .
\end{aligned}
$$

Collecting together the two criteria (9) and (10), we obtain

Theorem 3.2 Suppose that $m \geqslant 2, n \geqslant 4, n$ even. Then for any cube $\mathcal{B}$ centered at the origin, if (9), (10) hold, then

$$
\sum_{\mathbf{x} \in V} \alpha(\mathbf{x}) \geqslant \frac{1}{3} \frac{|\mathcal{B}|^{2}}{p^{m}} .
$$

In particular

$$
|V \cap(\mathcal{B}+\mathcal{B})| \geqslant \frac{|\mathcal{B}|}{3 p^{m}} .
$$


It remains to prove under the hypothesis of Theorem 3.2, the existence of primitive solutions of the congruence (1). Recall $\mathbf{x}$ is called primitive if $\operatorname{gcd}\left(x_{1}, \ldots, x_{n}, p\right)=1$. We shall write $p \mid \mathbf{x}$ for imprimitive points. Thus we have to prove

$$
\sum_{\mathbf{x} \in V} \alpha(\mathbf{x})>\sum_{\substack{\mathbf{x} \in V \\ p \mid \mathbf{x}}} \alpha(\mathbf{x})
$$

Corollary 3.1 Suppose $m \geqslant 2, n>m, n$ even, $p>2^{2(n+1) /(n-2)} 3^{2 /(n-2)}$ and $\mathcal{B}$ is a cube with $B>6^{1 / n} p^{(m / 2)+(m / n)}$. Then $\mathcal{B}+\mathcal{B}$ contains a primitive solution of $(2)$.

Proof. As in the proof of Lemma 3.1 with $j=m-1$, we have

$$
\begin{aligned}
\sum_{\substack{\mathbf{x} \in V \\
p \mid \mathbf{x}}} \alpha(\mathbf{x}) & =\sum_{\substack{p \mid x_{i}, 1 \leqslant i \leqslant n}} \alpha(\mathbf{x})<\sum_{\substack{\mathbf{u} \in B \\
\mathbf{u}+\mathbf{v} \equiv 0(\bmod p)}} \sum_{\mathbf{v} \in B} 1 \\
& \leqslant \prod_{i=1}^{n} B_{i}\left(\left[\frac{B_{i}}{p}\right]+1\right) \\
& =B^{n}\left(\left[\frac{B_{i}}{p}\right]+1\right)^{n} \\
& <\frac{B^{2 n}}{p^{n}}\left(1+\frac{p}{B}\right)^{n} \\
& \leqslant \frac{B^{2 n}}{p^{n}}(1+\varepsilon)^{n}
\end{aligned}
$$

where $\varepsilon<p^{-[(m / 2)+(m / n)]+1}$. However according to Theorem 3.2 we have,

$$
\sum_{\mathbf{x} \in V} \alpha(\mathbf{x}) \geqslant \frac{|\mathcal{B}|^{2}}{3 p^{m}}
$$

Hence it follows that

$$
\sum_{\substack{\mathbf{x} \in V \\ p \nmid \mathbf{x}}} \alpha(\mathbf{x}) \geqslant \frac{|\mathcal{B}|^{2}}{3 p^{m}}-\sum_{\substack{\mathbf{x} \in V \\ p \mid \mathbf{x}}} \alpha(\mathbf{x}) \geqslant \frac{|\mathcal{B}|^{2}}{3 p^{m}}-\frac{B^{2 n}}{p^{n}}(1+\varepsilon)^{n}>0,
$$

by our hypotheses on the size of $p$. This completes the proof.

\section{References}

[1] T. Cochrane, Small zeros of quadratic forms modulo p, J. Number Theory, 33, no.3 (1989), 286-292. 
[2] T. Cochrane, Small zeros of quadratic forms modulo p, II, Proceedings of the Illinois Number Theory Conference, (1989), Birkhauser, Boston (1990), 91-94

[3] T. Cochrane, Small zeros of quadratic forms modulo p, III, J. Number Theory, 33, no. 1 (1991), 92-99.

[4] T. Cochrane, Small zeros of quadratic congruences modulo pq, Mathematika, 37 (1990), no. 2, 261-272.

[5] T. Cochrane, Small zeros of quadratic congruences modulo pq, II, J. Number Theory 50 (1995), no. 2, 299-308.

[6] T. Cochrane and A. Hakami, Small zeros of quadratic congruences modulo $p^{2}$, II, Proceedings of the American Mathematical Society 140 (2012), no.12, 4041-4052.

[7] A. Hakami, Small zeros of quadratic congruences to a prime power modulus, PhD thesis, Kansas State University, 2009.

[8] A. Hakami, Small zeros of quadratic forms modulo $p^{2}$, JP J. Algera, Number Theory and Applications, 17,no. 2, (2011),141-162.

[9] A. Hakami, Small zeros of quadratic forms modulo $p^{3}$, J. Advanvces and Applications in Mathematical Sciences 9 (2011), no. 1, 47-69.

[10] A. Hakami, On Cochrane's estimate for small zeros of quadratic forms modulo p , Far East J. Math. Sciences,50 (2011), no. 2, 151-157.

[11] A. Hakami, Weighted quadratic partitions $\left(\bmod p^{m}\right)$, A new formula and new demonstration, Tamaking J. Math., 43, (2012), 11-19.

[12] L. Carlitz, Weighted quadratic partitions $\left(\bmod p^{r}\right)$, Math Zeitschr. Bd, 59, (1953), 40-46.

[13] D.R. Heath-Brown, Small solutions of quadratic congruences, Glasgow Math. J, 27 (1985), 87-93.

[14] D.R. Heath-Brown, Small solutions of quadratic congruences II, Mathematika, 38 (1991), no. 2, 264-284.

[15] A. Schinzel, H.P. Schlickewei and W.M. Schmidt, Small solutions of quadratic congruences and small fractional parts of quadratic forms, Acta Arithmetica, 37 (1980), 241-248.

[16] Y. Wang, On small zeros of quadratic forms over finite fields, Algebraic structures and number theory (Hong Kong, 1988), 269-274, World Sci. Publ., Teaneck, NJ, 1990. 
[17] Y. Wang, On small zeros of quadratic forms over finite fields, J. Number Theory, 31 (1989) 272-284.

[18] Y. Wang, On small zeros of quadratic forms over finite fields II, A Chinese summary appears in Acta Math. Sinica 37 No.5 (1994), 719-720. Acta Math. Sinica (N.S.) 9 no.4 (1993), 382-389.

Received: October, 2012 